\title{
Méthode d'identification numérique du Comportement Mécanique et Dynamique des Matériaux utilisés dans le Calcul Automobile
}

\author{
Numerical Method of identification of the mechanical and dynamic \\ behavior of materials used in automotive calculation
}

\author{
Abdelilah Elbazze ${ }^{1}$, Bouchaïb Radi ${ }^{2}$ \\ ${ }^{1}$ Laboratoire d'Ingénierie, Management Industriel et Innovation (IM2I) FSTS, Maroc elbazze.abdelilah@gmail.com \\ ${ }^{2}$ Laboratoire d'Ingénierie, Management Industriel et Innovation (IM2I) FSTS, Maroc, bouchaib.radi@yahoo.fr
}

RÉSUMÉ. Une modélisation cohérente et raisonnable du comportement du matériau et sa rupture sous les effets couplés de la déformation, de la vitesse de déformation et de la température par rapport à la contrainte d'écoulement du matériau est remarquablement cruciale afin de concevoir et d'optimiser les paramètres qui caractérisent le matériau numériquement et minimiser le maximum d'erreur entre la simulation et l'expérience. Le modèle de contrainte d'écoulement Johnson-Cook a été adopté pour modéliser et prédire le comportement de l'écoulement des matériaux à une vitesse de déformation et températures moyennes. De plus, le travail qui a été fait dans cet article est d'expliquer chaque phase d'identification des paramètres du modèle construit et comment examiné systématiquement par rapport aux données expérimentales. Ensuite, pour prédire le comportement d'endommagement aux matériaux, le modèle de rupture proposé par Johnson et Cook a été utilisé pour déterminer les paramètres de rupture du modèle, en se basant au début sur la détermination de la triaxialité pour une température ambiante et sous une condition de vitesse de déformation quasi-statique. La méthode expliquée dans ce travail est la plus utilisée dans l'industrie automobile pour caractériser les matériaux appropries aux calculs numériques type choc.

ABSTRACT. A coherent and reasonable modeling behavior of the material and its damage under the coupled effects of the deformation, the strain rate and the temperature compared to the stress of flow of the material is remarkably crucial in order to design and optimize the parameters that characterize the material numerically and minimize the maximum error between simulation and experiment. The Johnson-Cook flow constraint model was adopted to model and predict the behavior of material flow at strain rate and temperature average. In addition, the work that has been done in this article is to explain each phase of identification of the parameters of the constructed model and how it is systematically examined in relation to the experimental data. Then, to predict the behavior of damage, the failure model proposed by Johnson and Cook was used to determine the parameters of rupture of the model, based initially on the determination of the triaxiality for an ambient temperature and under a quasi-static strain rate condition. The method explained in this work is the most used in the automotive industry to characterize suitable materials for numerical crash calculation.

MOTS-CLÉS. Matériaux numériques, modélisation, loi de Johnson Cook, endommagement, automobile, triaxialité, crash. KEYWORDS. Numerical materials, modeling, Johnson Cook's law, damage, automotive, triaxiality, crash.

\section{Introduction}

Modéliser le comportement des matériaux et les phénomènes d'endommagement causés par la déformation plastique dans les matériaux soumises à des sollicitations de traction où flexion, est pour un objectif de réduire le coût et le temps des expériences. Dans la pratique industrielle comme l'automobile, le modèle de matériau et de dommages de Johnson Cook (JC) est largement intégré dans la plupart des outils d'éléments finis (EF) disponibles pour modéliser le comportement des matériaux, de sa capacité à prédire les paramètres du modèle avec moins d'effort. Il est primordiale que le modèle de contrainte d'écoulement proposé, bien conçu et fiable, est plus favorable. Même si les modèles de contrainte d'écoulement sont divisés en différentes catégories, telles que les modèles physiques, empiriques et semi-empiriques, l'objectif de ces modèles est d'obtenir une prédiction précise du comportement du matériau, pour un matériau spécifique reste le même [HE 18]. Le développement d'un modèle de contrainte d'écoulement approprié pour le calcul automobile est essentiel pour prédire le comportement de déformation du matériau à des vitesses de déformation et des températures (c) 2020 ISTE OpenScience - Published by ISTE Ltd. London, UK - openscience.fr 
élevées, par conséquent, des recherches raisonnables ont été effectuées en considérant divers matériaux et méthodes de modélisation.

Aviral Shrot et al. [SHR 12] ont proposé une méthode utilisant l'algorithme de recherche de Levenberg-Marquardt pour l'identification inverse des paramètres des matériaux JC. Un ensemble de paramètres JC a été utilisé pour développer un modèle EF idéalisé pour le processus d'usinage. Ensuite, la méthode d'identification inverse a été utilisée pour estimer le JC en examinant la morphologie des copeaux et la force de coupe pendant le processus. Ils ont conclu qu'il est possible de ré-identifier les paramètres du modèle par des méthodes inverses; cependant, les paramètres estimés à partir des résultats de la simulation étaient presque identiques à l'ensemble d'origine.

À partir d'une étude de la littérature, elle a identifiée que la construction d'un modèle EF approprié nécessite généralement un effort expérimental coûteux, et une modélisation appropriée du comportement et l'endommagement du matériau en prenant compte à la fois le début et l'évolution des ruptures. Même si de nombreux chercheurs ont travaillé sur le modèle JC d'origine, très peu de chercheurs ont été signalés sur la stratégie d'identification et d'optimisation des constantes du modèle JC pour améliorer la prévisibilité du modèle. De plus, jusqu'à présent, il n'y a eu aucune tentative de développement d'un matériau JC détaillé et d'un modèle de dommages pour les matériaux en acier au carbone. Le but de ce travaille est d'identifier les paramètres du modèle JC constitutif et son endommagement le plus cohérent.

\section{Méthode d'identification numérique de la loi de comportement des matériaux}

\subsection{Modèle de Johnson Cook}

La relation entre la contrainte et la déformation dans les matériaux métalliques peut être décrite par le modèle Johnson-Cook; dans des conditions de grande déformation, de vitesse de déformation élevée et de températures élevées. Étant sous une forme simple et comme il nécessite moins d'efforts pour estimer les constantes des matériaux [ABB 14-SAM 09], il a été largement utilisé par des constructeurs automobile et chercheurs pour prédire le comportement des matériaux en simulation ; dans le cas d'étude de choc (grande déformation). Le modèle de contrainte (JC) est exprimé comme suit :

$$
\sigma=\left(A+B \varepsilon^{n}\right)\left(1+C \ln \dot{\varepsilon}^{*}\right)\left(1-T^{*} m\right)
$$

Où $\sigma$ est la contrainte équivalente et $\varepsilon$ la déformation plastique équivalente. Les constantes matérielles sont $\mathrm{A}, \mathrm{B}, \mathrm{n}, \mathrm{C}$ et $\mathrm{m}$. A est la limite élastique du matériau dans les conditions de référence, $\mathrm{B}$ est la constante d'écrouissage, $\mathrm{n}$ est le coefficient d'écrouissage, $\mathrm{C}$ est le coefficient de renforcement de la vitesse de déformation et m est le coefficient d'adoucissement thermique [CAO 14].

Les trois composants entre parenthèses de l'équation [1] représentent, de gauche à droite, l'effet de durcissement par déformation, l'effet de renforcement de la vitesse de déformation et l'effet de la température, qui influencent les valeurs de contrainte [MUR 17]. Dans le modèle de contrainte d'écoulement, $\dot{\varepsilon}^{*}$ et $\mathrm{T}^{*}$ sont :

$$
\dot{\varepsilon}^{*}=\frac{\dot{\varepsilon}}{\dot{\varepsilon}_{\mathrm{ref}}}, T^{*}=\frac{T-T_{r}}{T_{m}-T_{r}}
$$

où $\dot{\varepsilon}^{*}$ est la vitesse de déformation sans dimension, $\mathrm{T}^{*}$ est la température sans dimension, $\mathrm{T}_{\mathrm{m}}$ est la température de fusion du matériau et $\mathrm{T}$ est la température de déformation. $\dot{\varepsilon}_{\text {ref }}$ et $\mathrm{T}_{\text {ref }}$ sont respectivement la vitesse de déformation de référence et la température de référence [MUR 17]. Dans cet article, on considère que les conditions expérimentales, de la vitesse de déformation de 
référence $\dot{\varepsilon}_{\text {ref }}$, et la température de référence $\mathrm{T}_{\text {ref, }}$ ont été prises respectivement à $1223 \mathrm{~K}$ et $1 \mathrm{~s}^{-1}$. Conformément aux conditions de référence, la constante de matériau, $\mathrm{A}$, a été déterminée comme étant de 50,103 MPa pour d'autres calculs.

\subsubsection{Détermination des constantes $B$ et $n$}

Lorsque la température de déformation est $\mathrm{T}=\mathrm{T}_{\text {ref }}$ et que la vitesse de la déformation $\dot{\varepsilon}=\dot{\varepsilon}$ ref, l'équation (1) est modifiée comme suit [MUR 17]:

$$
\sigma=\left(A+B \varepsilon^{n}\right)
$$

Ici, l'impact de la vitesse de déformation et l'effet de ramollissement thermique sont négligées. En réarrangeant l'équation [2] et en prenant le logarithme naturel des deux côtés de l'équation [2], l'équation modifiée peut être obtenue comme indiqué ci-dessous [MUR 17] :

$$
\ln (\sigma-A)=n \ln \varepsilon+\ln B
$$

En substituant les valeurs de contrainte d'écoulement et de la déformation aux conditions de déformation de référence dans l'équation [3], la relation linéaire entre $\ln (\sigma-A)$ et $\ln \varepsilon$ a été tracé, puis le modèle de régression du premier ordre sera utilisé pour ajuster les données comme le montre la figure 1. D'après la figure 1 , il est à noter que plus de $96 \%$ des données se trouvent très près de la droite de régression, ce qui montre la meilleure prévisibilité de la distribution des données. Par conséquent, les constantes matérielles $\mathrm{B}$ et $\mathrm{n}$ ont été estimées à partir de la pente et de l'ordonnée à l'origine de la courbe ajustée.

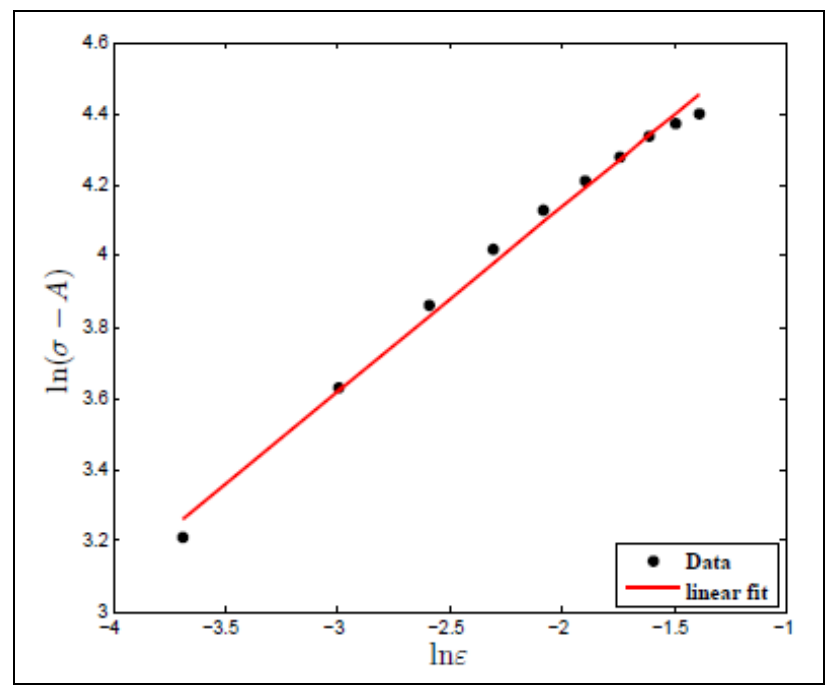

Figure 1. Exemple de courbe qui montre la relation entre la contrainte d'écoulement et la déformation pour identifier les deux paramètres B et n. [MUR 19]

\subsubsection{Détermination de la constante $C$}

Lorsque la température de déformation $\mathrm{T}=\mathrm{T}_{\text {ref }}$, l'équation [1] peut être remodelée comme indiqué ci-dessous [MUR 17]:

$$
\sigma=\left(A+B \varepsilon^{n}\right)\left(1+C \ln \dot{\varepsilon}^{*}\right)
$$

tandis que les effets de ramollissement thermique sont ignorées. Réarranger l'équation [4] entraînera la forme suivante: 


$$
\frac{\sigma}{\left(A+B \varepsilon^{n}\right)}=\left(1+C \ln \dot{\varepsilon}^{*}\right)
$$

Initialement, les valeurs des constantes matérielles $\mathrm{A}, \mathrm{B}$ et $\mathrm{n}$, obtenues à la section 1.1.1, ont été substituées dans l'équation [5]; puis, $\_\frac{\sigma}{-} \approx C \ln \tilde{\varepsilon}^{*}$ sera tracé comme une courbe, comme le montre la $\left(A+B \varepsilon^{n}\right)$

figure 2. Par la suite, l'ajustement linéaire sera effectué en utilisant le modèle de régression du premier ordre avec une valeur d'interception de 1, en considérant l'écoulement de contrainte décrit pour chaque valeur de la vitesse de déformation permet de tracer la pente de la courbe d'ajustement, la valeur constante du matériau, $\mathrm{C}$, sera estimée à partir de cette pente. Ici, il est important de mentionner qu'au début, la constante de matériau, $\mathrm{C}$, a été estimée sur la base de la méthode traditionnelle en tenant compte de toutes les valeurs de déformation. Cependant, dans cette recherche, la procédure d'optimisation a été adoptée pour trouver la valeur $\mathrm{C}$ optimale afin de réduire l'erreur de prédiction par rapport aux données expérimentales.

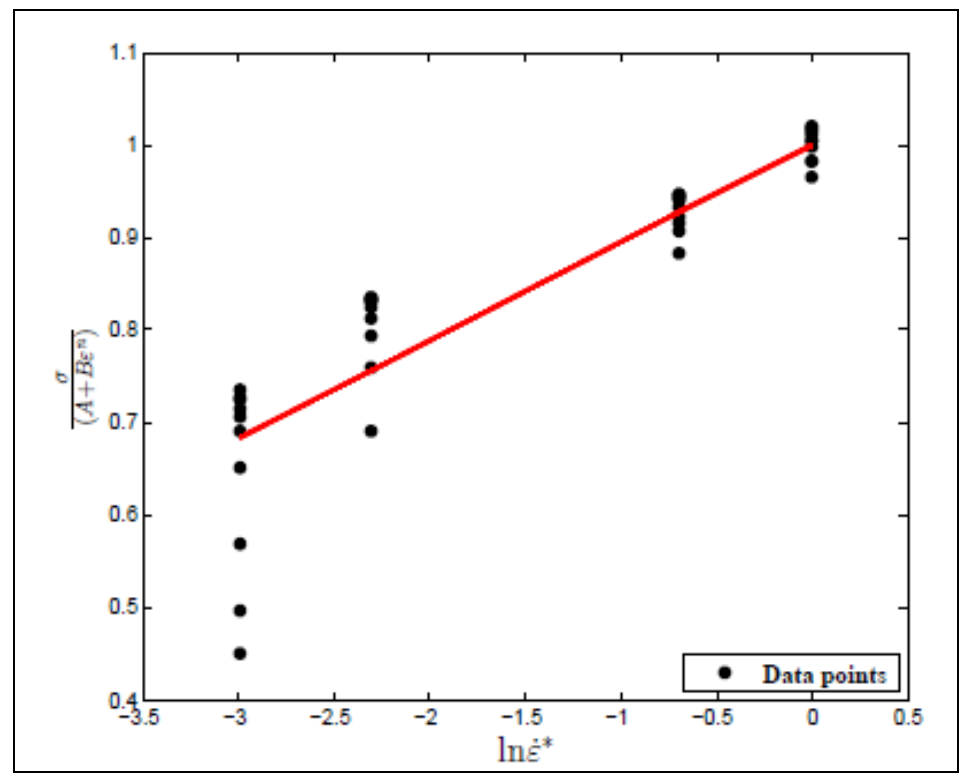

Figure 2. Exemple de courbe $\frac{\sigma}{\left(A+B \varepsilon^{n}\right)}$ et $\ln \dot{\varepsilon}^{*}$. [MUR 19]

\subsubsection{Détermination de la constante $m$}

Lorsque la vitesse de déformation est égale à la vitesse de déformation de référence $\dot{\varepsilon}=\dot{\varepsilon}_{\text {ref }}$, l'équation [1] peut être simplifiée comme [MUR 17] :

$$
\sigma=\left(A+B \varepsilon^{n}\right)\left(1-T^{*} m\right)
$$

Ici, les influences de l'effet de renforcement de la vitesse de déformation sont négligées. Equation [6] est réorganisée sous la forme suivante :

$$
1-\frac{\sigma}{\left(A+B \varepsilon^{n}\right)}=T^{* m}
$$

En prenant le logarithme naturel des deux côtés de l'équation [7], l'équation suivante peut être obtenue comme: 


$$
\ln \left[1-\frac{\sigma}{\left(A+B \varepsilon^{n}\right)}\right]=m \ln \left(T^{*}\right)
$$

En substituant les valeurs des constantes matérielles A, B et $\mathrm{n}$ dans l'équation [8]et en ajustant les points des données en utilisant le modèle de régression du premier ordre, comme le montre la figure 3 , la constante matérielle $\mathrm{m}$ sera déterminée à partir de la pente de la courbe ajustée en considérant la méthode conventionnelle. Comme pour la constante de matériau $\mathrm{C}$, et à des fins d'optimisation. Ciaprès, une procédure d'optimisation contrainte aux limites (figure 4) sera utilisée pour trouver la solution optimale des constantes de matériau $\mathrm{C}$ et $\mathrm{m}$, et la formulation d'optimisation utilisée est exprimée ci-dessous :

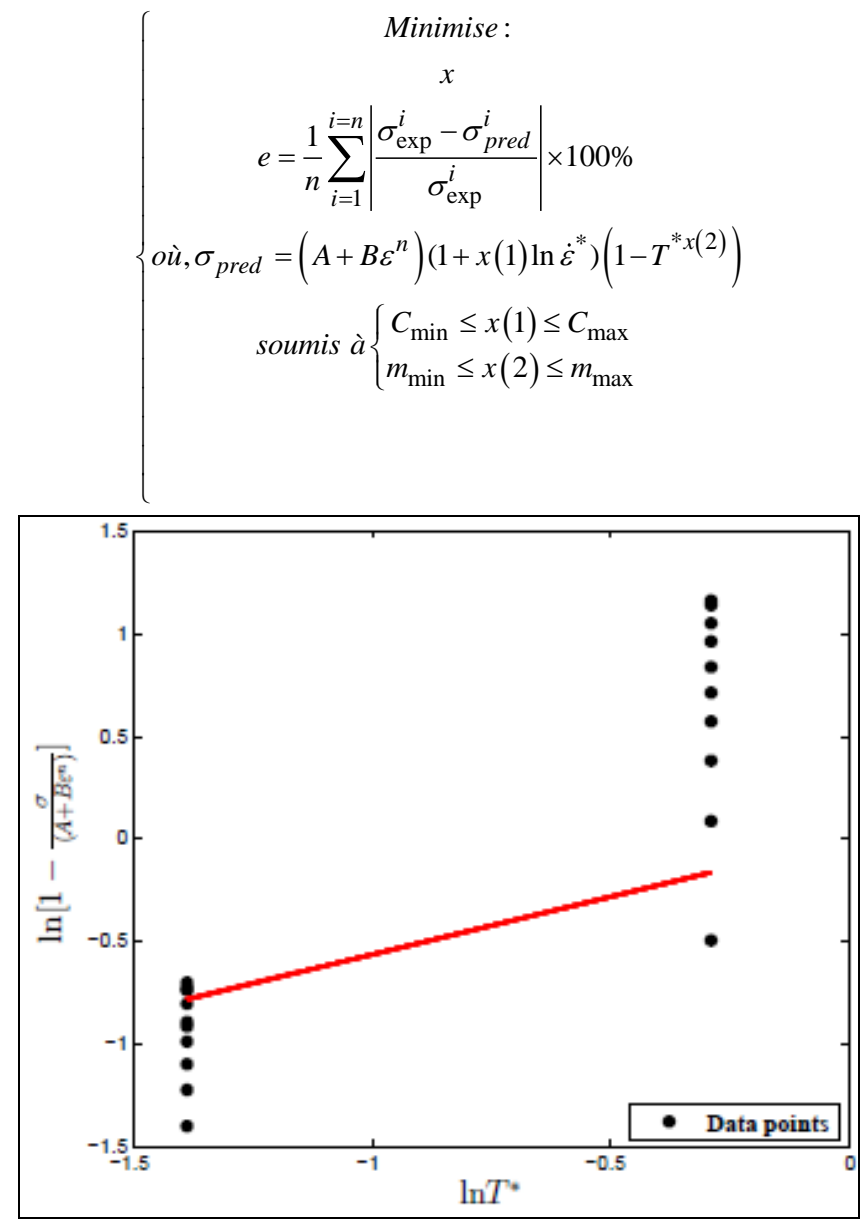

Figure 3. Exemple de calcul qui montre la relation entre $\ln \left[1-\frac{\sigma}{\left(A+B \varepsilon^{n}\right)}\right]$ et $\ln T^{*}$ sous les conditions de référence. [MUR 19]

Pour résoudre le problème d'optimisation, un solveur de programmation non linéaire, peut être utilisé avec l'algorithme du point intérieur (IP) pour minimiser l'erreur relative absolue moyenne entre les données expérimentales et les données prédites. La principale raison d'utiliser l'algorithme IP était de trouver le minimum d'une fonction objective en présence de contraintes liées seules. 


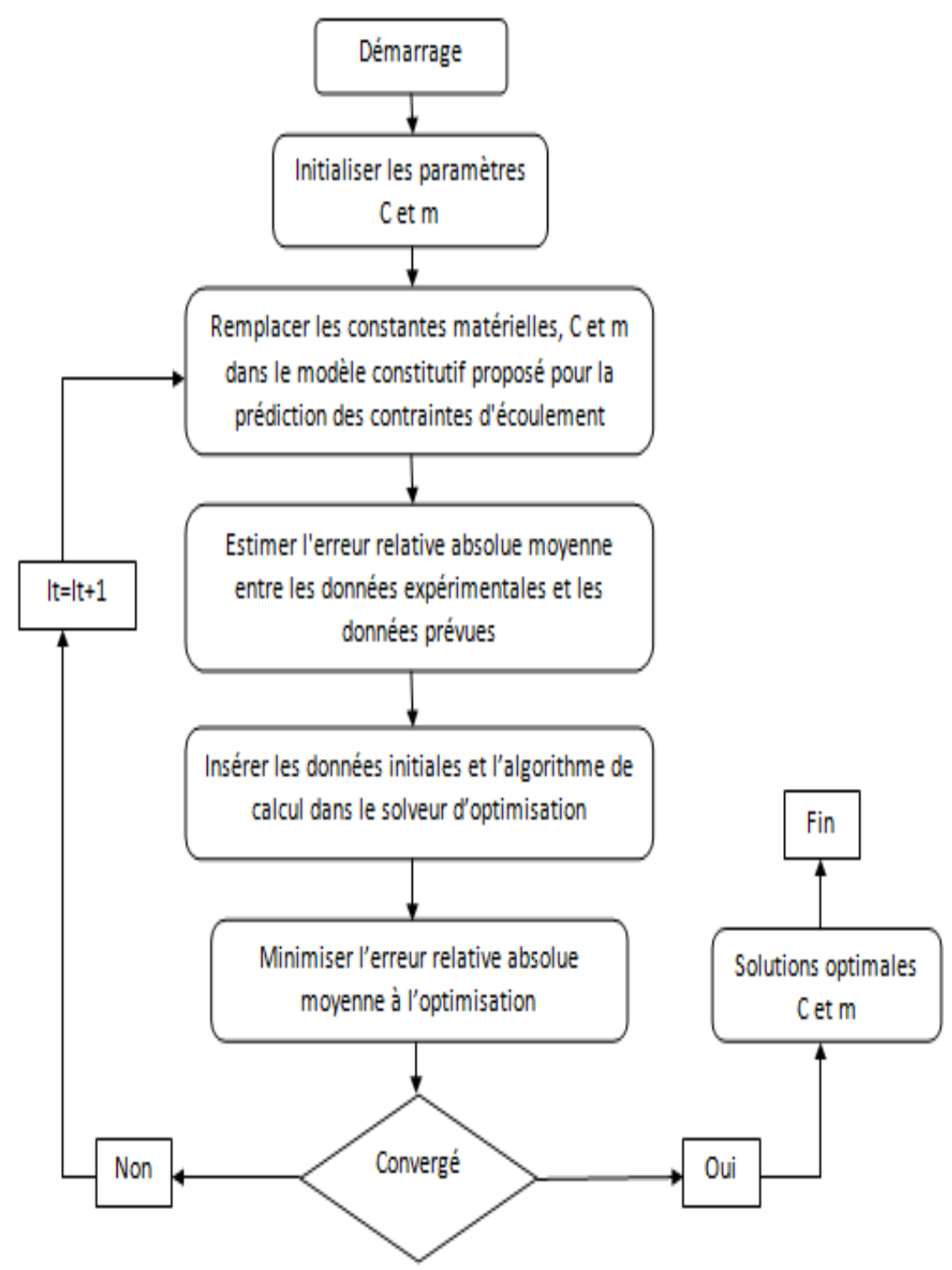

Figure 4. Organigramme de la procédure d'optimisation pour trouver le minimum d'une fonction objectif en présence de contraintes liées. [MUR 19]

\section{Méthode d'identification numérique de la loi de rupture des matériaux}

Pour mieux représenter les phénomènes de rupture, et l'évolution de l'endommagement dans les matériaux. La triaxialité nous permet d'identifier les tubes de chargement soumis à l'éprouvette au cours du test de traction ou flexion. Dans ce travail, nous avons reproduit numériquement les mêmes conditions d'essai de traction, dans l'objectif est de tracer la courbe de triaxialité et identifier les paramètres de rupture $\sum \mathrm{D}_{\mathrm{i}}$ en se basant de la loi de rupture de Johnson Cook.

Johnson et Cook ont proposé que la déformation à la rupture dépende généralement du rapport de triaxialité des contraintes, de la vitesse de déformation et de la température. Le modèle de fracture JC peut s'écrire comme suit [BAN 15]:

$$
\varepsilon_{\mathrm{f}}=\left[\mathrm{D}_{1}+\mathrm{D}_{2} \exp \left(\mathrm{D}_{3}\left(\frac{\sigma_{\mathrm{m}}}{\sigma_{\mathrm{eq}}}\right)\right)\right]\left[1+\mathrm{D}_{4} \ln \left(\dot{\varepsilon}_{\mathrm{p}}^{*}\right)\right]\left[1+\mathrm{D}_{5} \mathrm{~T}^{*}\right]
$$

où $\mathrm{D}_{1}$ à $\mathrm{D}_{5}$ sont les constantes du modèle d'endommagement, $\sigma_{\mathrm{m}}$ est la contrainte moyenne et $\sigma_{\mathrm{eq}}$ est la contrainte équivalente [MUR 17]. L'endommagement d'un élément est défini sur la base d'une loi de dommage cumulatif, et il peut être représenté de manière linéaire comme indiqué ci-dessous [BUZ 15MUR 17] : 


$$
D=\sum\left(\frac{\Delta \varepsilon}{\varepsilon_{f}}\right)
$$

où $\Delta \varepsilon$ est l'incrément de déformation plastique équivalent et $\varepsilon_{\mathrm{f}}$ est la déformation équivalente à la rupture dans les conditions actuelles de contrainte, de la vitesse de déformation et de la température.

$\mathrm{T}^{*}$ est la température non dimensionnelle, $\dot{\varepsilon}_{p}^{*}$ la vitesse de déformation plastique sans dimension qui peut s'écrire comme suit

$$
\dot{\varepsilon}_{p}^{*}=\frac{\dot{\varepsilon}}{\dot{\varepsilon}_{0}}, T^{*}=\frac{T-T_{r}}{T_{m}-T_{r}}
$$

où $\mathrm{T}_{\mathrm{m}}, \mathrm{T}_{\mathrm{r}}$ sont respectivement la température de fusion et la température ambiante et la vitesse de déformation initiale $\dot{\varepsilon}_{0}$. En raison de la rupture, la résistance du matériau diminue pendant la déformation, et la relation constitutive de contrainte pour l'évolution de l'endommagement peut être exprimée comme [BAN 15]:

$$
\sigma_{D}=(1-D) \sigma_{e q}
$$

Dans l'équation [11], $\sigma_{\mathrm{D}}$ est l'état de contrainte endommagé et $\mathrm{D}$ est le paramètre de dommage $(0<\mathrm{D}<1)$. De plus, la triaxialité des contraintes [BAI 08-BAO 05] et la contrainte équivalente peuvent être obtenues à partir de matériaux non endommagés compte tenu du comportement plastique jusqu'à la formation du rétrécissement [MUR 17]. Au départ, l'effet de la température et de la vitesse de déformation est considéré comme négligeable (température ambiante et vitesse de déformation quasi statique) dont l'objectif est d'étudier l'effet de la triaxialité sur l'évolution du phénomène d'endommagement. Noté bien qu'il faut reproduire les mêmes conditions d'essai sur des éprouvettes dans la modélisation numérique en utilisant les résultats expérimentaux comme données d'entrée pour la caractérisation numérique du matériau. Avant de tracer les courbes de triaxialité, les résultats numériques doivent être vérifiés avec les observations expérimentales en terme de contrainte /déformation pour la plage élastique et plastique jusqu'au rétrécissement, comme le montre la figure 5.

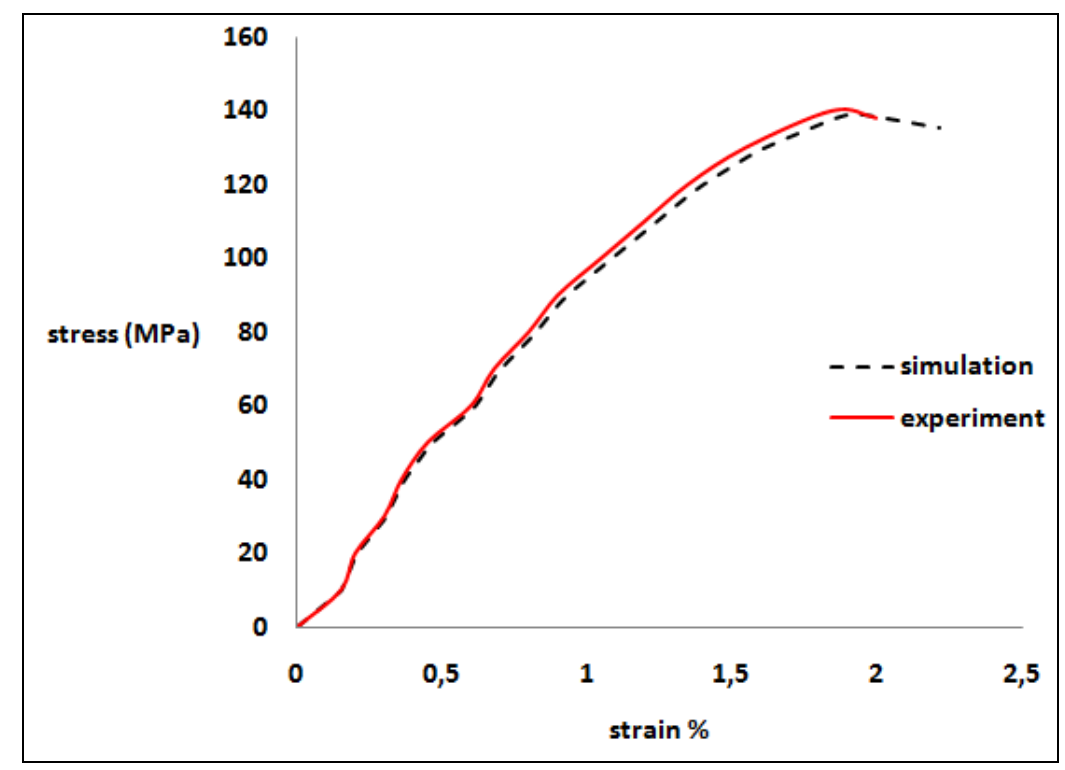

Figure 5. Exemple de contrainte en fonction de la déformation

De la figure 5, il est évident que l'estimation des propriétés mécaniques des expériences a été parfaitement effectuée et peut en outre être utilisé pour effectuer l'estimation de la triaxialité des contraintes sans aucune barrière. Par la suite, les composantes de contrainte, telles que $\sigma_{1}, \sigma_{2}$ et $\sigma_{3}$, sont calculées à partir de la moyenne entre les éléments qui se brisent dans l'échantillon. Ainsi, les composantes de contrainte ont été substituées dans l'équation [12] pour déterminer la contrainte 
moyenne $\sigma_{\mathrm{m}}$, et la contrainte équivalente $\sigma_{\mathrm{eq}}$, et l'ensemble des triaxialités de contrainte estimées pour un échantillon entier est illustré à la figure 6.

$$
\sigma^{*}=\frac{\left(\sigma_{1}+\sigma_{2}+\sigma_{3}\right)}{3 * \sqrt{0,5^{*}\left[\left(\sigma_{1}-\sigma_{2}\right)^{2}+\left(\sigma_{2}-\sigma_{3}\right)^{2}+\left(\sigma_{1}-\sigma_{3}\right)^{2}\right]}}
$$

Réarrangeant de l'équation [9] en négligeant les effets de la vitesse de déformation et de la température, l'équation du modèle de rupture ne peut être réécrite qu'en termes d'effet de triaxialité des contraintes par rapport à la déformation de rupture comme suit [MUR 17] :

$$
\varepsilon_{f}=D_{1}+D_{2} \exp \left(D_{3} \sigma^{*}\right)
$$

En substituant les triaxialités de contrainte et les valeurs de déformation de rupture correspondantes dans l'équation [13], le tracé de la relation $\varepsilon_{f}=\sigma^{*}$ a été développé à partir de la courbe de triaxialité, comme le montre la figure 6 , les paramètres du modèle $\mathrm{D}_{1}, \mathrm{D}_{2}$ et $\mathrm{D}_{3}$ seront calculés ultérieurement.

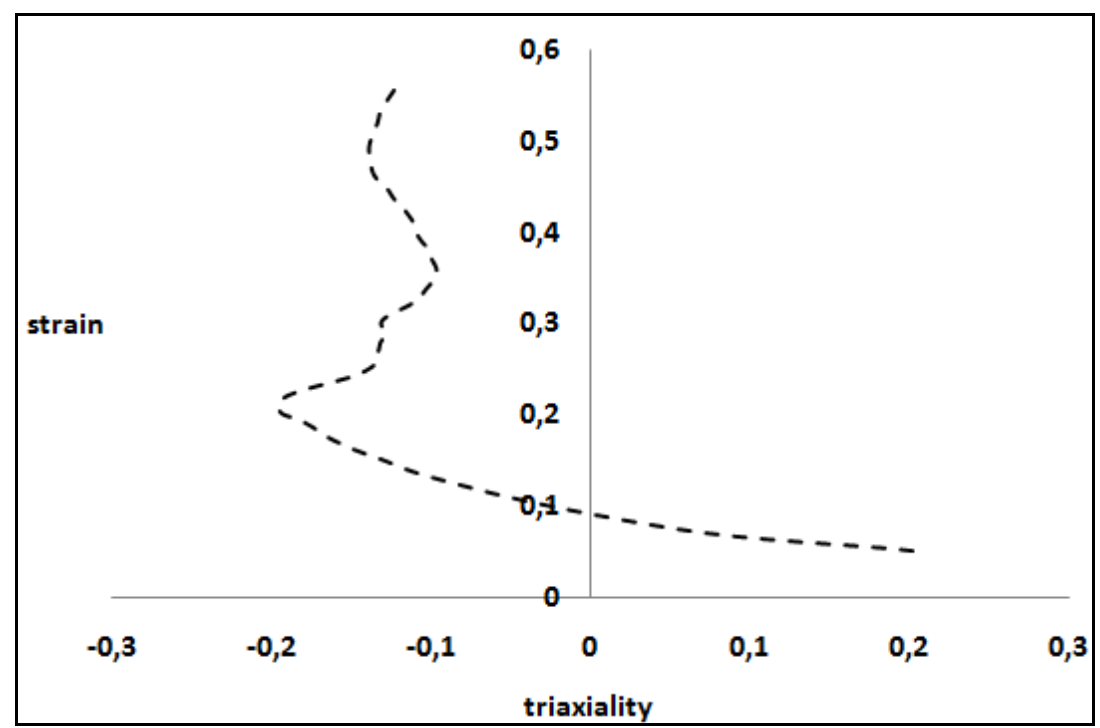

Figure 6. Exemple de courbe de déformation à la rupture en fonction de la contrainte de triaxialité.

Par la suite, les deux autres paramètres de l'endommagement $\mathrm{D}_{4}$ et $\mathrm{D}_{5}$ dépendants de la vitesse de déformation et de la température, D4 et D5 seront obtenus à partir de deux ensembles de données de température et de vitesse de déformation élevées en interprétant la variation de déformation à la rupture [MUR 17]. Les paramètres estimés du modèle de fracture JC peuvent être utilisés dans les calculs automobile.

\section{Conclusion}

Dans cet article, le travail qui a été fait est de montrer la méthodologie d'identification des paramètres du modèle Johnson-Cook de matériau et sa rupture, La procédure d'optimisation pour minimiser l'erreur de prédiction entre les expériences et la simulation afin d'améliorer la capacité du modèle constitutif JC et sa rupture pour modéliser mieux les matériaux. Dans l'ensemble, la méthode proposée dans ce travail est le plus simple et qui donne une bonne approximation du comportement des matériaux en numérique. Cette méthode est le plus utilisée dans l'automobile surtout pour caractériser les matériaux qui seront utilisés pour faire des calculs type choc. 


\section{Bibliographie}

[HE 18] HE J., CHEN F.,WANG B., ZHU L.B., «A modified Johnson-Cook model for $10 \% \mathrm{Cr}$ steel at elevated temperatures and a wide range of strain rates», Mater. Sci. Eng, 715, 1-9, 2018.

[SHR 12] SHROT A., BÄKER M., « Determination of Johnson-Cook parameters from machining simulations. Computer», Mater. Sci, 52, 298-304, 2012.

[MIL 09] MILANI A.S., DABBOUSSI W., NEMES J.A., ABEYARATNE R.C., «An improved multi-objective identification of Johnson-Cook material parameters», Int. J. Impact Eng, 36, 294-302, 2009.

[MAJ 11] MAJZOOBIA G.H., DEHGOLAN F.R., «Determination of the constants of damage models», Procedia Eng., 10, 764-773,2011.

[BAN 15] BANERJEE A., DHAR S., ACHARYYA S., DATTA D., NAYAK N., « Determination of Johnson cook material and failure model constants and numerical modelling of Charpy impact test of armour steel», Mater. Sci. Eng., 640, 200-209, 2015.

[BUZ 15] BUZYURKINA A.E., GLADKYB I.L., KRAUSA E.I., «Determination and verification of Johnson-Cook model parameters athigh-speed deformation of titanium alloys » Aerosp. Sci. Technol., 45, 121-127., 2015.

[ABB 14] ABBASI-BANI A., ZAREI-HANZAKI A., PISHBIN M.H., HAGHDADI N., «A comparative study on the capability of Johnson-Cook and Arrhenius-type constitutive equations to describe the flow behavior of Mg-6Al1Zn alloy», Mech. Mater., 71, 52-61., 2014.

[SAM 09] SAMANTARAY D., MANDAL S., BHADURI A.K., «A comparative study on Johnson Cook, modified Zerilli-Armstrong and Arrhenius-type constitutive models to predict elevated temperature flow behaviour in modified 9Cr-1Mo steel», Comput. Mater. Sci., 47, 568-576., 2009.

[CAO 14] CAO Y., DI H.S., MISRA R.D.K., ZHANG J., « Hot Deformation Behavior of Alloy $800 \mathrm{H}$ at Intermediate Temperatures: Constitutive Models and Microstructure Analysis », J. Mater. Eng. Perform., 23, 42984308.,2014.

[MUR 17] MURUGESAN M., LEE S., KIM D., KANG Y.H., KIM N., « A Comparative Study of Ductile Damage Models Approaches for Joint Strength Prediction in Hot Shear Joining Process» , Procedia Eng., 207, 16891694.,2017.

[BAI 08] BAI Y., WIERZBICKI T., « A new model of metal plasticity and fracture with pressure and Lode dependence», Int. J. Plast., 24, 1071-1096., 2008.

[BAO 05] BAO Y., " Dependence of ductile crack formation in tensile tests on stress triaxiality, stress and strain ratios », Eng. Fract. Mech., 72, 505-522., 2005.

[MUR 19] MURUGESAN M., JUNG D W., «Johnson Cook Material and Failure Model Parameters Estimation of AISI-1045 Medium Carbon Steel for Metal Forming Applications», Materials, 12, 609., 2019. 